pag

Business School

WORKING PAPER SERIES

Working Paper

2014-262
Les normes IFRS ont-elles amélioré le contenu informationnel des immatériels? Le cas des entreprises françaises cotées

Sandrine Boulerne

Jean-Michel Sahut

http://www.ipag.fr/fr/accueil/la-recherche/publications-WP.html

IPAG Business School

184, Boulevard Saint-Germain

75006 Paris

France 


\title{
Les normes IFRS ont-elles amélioré le contenu informationnel des immatériels ? Le cas des entreprises françaises cotées
}

\author{
Sandrine Boulerne \\ Maitre de conférences, IAE de TOURS (CERMAT-EA2109) \\ 50 Avenue Jean Portalis 37206 Tours Cedex 03 \\ Enseignant chercheur à l'ESCEM \\ 1 rue Léo Delibes - BP 53537205 Tours Cedex 3 \\ sandrine.boulerne@univ-tours.fr \\ Jean-Michel Sahut \\ Enseignant chercheur à l'IPAG Business School \\ 184 Bd Saint Germain, 75007 Paris
}

\section{Résumé}

Les normes IAS/IFRS sont présumées fournir une information plus uniforme, pertinente et reflétant mieux la situation financière et le patrimoine de l'entreprise. Dans cet article, nous étudions le contenu informationnel des actifs incorporels selon les normes IAS/IFRS par rapport au PCG pour les entreprises du SBF 250. Nous montrons d'une part que le passage aux IAS/IFRS n'a pas fait varier le montant global des actifs incorporels, même s'il s'est opéré des effets de substitution au profit du goodwill, d'autre part l'existence d'une plus grande pertinence des informations véhiculées par les données comptables des actifs incorporels totaux et du goodwill lorsqu'ils sont valorisés selon les normes IAS/IFRS. Avec ces normes, les marchés financiers intègrent mieux de telles contributions dans les cours et les rentabilités boursières, surtout pour les firmes ayant une haute densité en actifs incorporels.

\begin{abstract}
Have IFRS improved the information content of intangible? The case of French companies listedIAS/IFRS are presumed to provide more uniform and relevant information which reflect the financial position and the assets of firm. In this article, we are studying the information content of intangible assets according to IAS/IFRS compared to the French GAAP for French listed firms (SBF 250 index). We show that, on one hand that the transition to IAS/IFRS did not change the overall amount of intangible assets, even though it operated substitution effects in favour of goodwill, on the other hand the greater accounting relevance of total intangible assets and goodwill when they are valued according to IAS/IFRS. With these standards, financial markets better integrate such contributions in stock market price and returns, especially for firms with a high density of intangible assets.
\end{abstract}

Post Print : Boulerne Sandrine, Sahut Jean-Michel, «Les normes IFRS ont-elles amélioré le contenu informationnel des immatériels? Le cas des entreprises françaises cotées », Comptabilité - Contrôle - Audit 1/ 2010 (Tome 16), p. 7-7 


\section{Introduction}

$\mathrm{L}$ nature des investissements effectués par les entreprises a singulièrement changé au cours des dernières années. L'évolution des pratiques des entreprises et de leur environnement économique ont fait émerger de nouvelles variantes des fonctions de production dans lesquelles la part de l'immatériel comme facteur d'une part, et comme produit d'autre part, est une fonction croissante (De Montmorillon, 2001). La globalisation des marchés financiers, le développement de l'économie des connaissances et le nombre grandissant de fusions et acquisitions représentent, dans ce cadre, les facteurs de changement les plus fréquemment cités. Ce nouveau contexte a contribué à l'accroissement des valeurs immatérielles des entreprises tels que les marques, brevets, frais de formation, frais de recherche et développement, compétences organisationnelles, etc. Face à ce flux grandissant d'incorporels, les systèmes normatifs comptables traditionnels, reposant sur un «principe transactionnel », éprouvent de plus en plus de difficulté à remplir leur rôle informatif d'aide à la décision (Lev, 1999). Par ailleurs, le problème de comparabilité de l'information comptable demeure difficile particulièrement en Europe, avant 2005, compte tenu de la diversité des choix et conditions de transcription comptable des investissements incorporels offerts par les différents.

Les normes IAS/IFRS, obligatoires depuis le $1^{\text {er }}$ janvier 2005 en Europe pour les sociétés cotées, visent à donner une information plus uniforme, pertinente et reflétant mieux la situation financière patrimoniale des entreprises que les normes locales qu'elles utilisaient auparavant [1] Preliminary Views on an improved Conceptual Framework... [1]. Cet objectif des normes IAS/IFRS est d'ailleurs mis en exergue par la Commission européenne [2] «Sa nouvelle structure organisationnelle devrait être... [2]. De plus, certains chercheurs suggèrent que ces normes améliorent le contenu informatif des données comptables (Schipper, 2005), notamment au travers de l'étude des firmes suisses, allemandes et autrichiennes qui les ont adoptées de manière anticipée (Dumontier et Maghraoui, 2007 ; Affes et Callimaci, 2008). Toutefois, d'autres études montrent qu'il est difficile de prévoir l'impact du changement de certaines règles comptables sur la qualité des données comptables du fait que cette dernière 
est influencée par plusieurs facteurs institutionnels complexes (Ball et alii, 2003 ; Ding et alii, 2007). En effet, les règles comptables existent dans une mosaïque avec d'autres règles institutionnelles. Changer un élément de cette mosaïque n'est pas toujours la solution lorsque les autres éléments demeurent invariants (Hope et alii, 2006 ; Ding et alii, 2007).

Si les études déjà réalisées, sur le marché français, ont déjà permis de montrer un impact relativement modéré des normes IAS/IFRS sur les capitaux propres (Schatt et Gross, 2007), ou les immobilisations corporelles (Benabdellah et Teller, 2006), de nombreuses questions restent posées quant à leur incidence sur les actifs incorporels, lesquels déterminent aussi bien la performance que la valorisation des firmes et en particulier de celles intensives en R\&D (Lantz et Sahut, 2005). De plus, la faible taille des échantillons considérés dans ces études, en général les entreprises du CAC 40 (Schatt et Gross, 2007 ; Bessieux-Ollier et Walliser, 2007), limite la portée de leurs conclusions.

Dans le référentiel international, les immobilisations incorporelles sont régies par l'IAS 38 [3] Cette norme a été approuvée en juillet 1998 et révisée... [3] et l'IFRS 3 en cas de regroupement d'entreprise.

L'IAS 38 définit une immobilisation incorporelle comme étant «un actif non monétaire identifiable sans substance physique. Un actif répond au critère d'identifiabilité dans la définition d'une immobilisation incorporelle lorsqu'il est séparable [4] Actif qui peut être vendu sans céder d'autres éléments... [4], ou s'il résulte de droits contractuels ou d'autres droits légaux, que ces droits soient ou non cessibles ou séparables de l'entité ou d'autres droits et obligations » (IAS 38, § 12).

Elle impose la comptabilisation de toute dépense immatérielle en immobilisation incorporelle si, et seulement si (IAS 38, § 21) :

1. il est probable que les avantages économiques futurs attribuables à l'actif iront à l'entité,

2. le coût de cet actif peut être évalué de façon fiable.

Une dépense pour un investissement incorporel doit donc soit être comptabilisée en charge soit être capitalisée. Le traitement optionnel n'existe pas. Si cette dépense résulte d'un regroupement d'entreprise et ne peut pas être comptabilisée en tant qu'immobilisation incorporelle, alors elle est incorporée au montant attribué au goodwill à la date d'acquisition. 
Ainsi plusieurs dépenses pour des investissements incorporels (comme la publicité, les frais de recherche, les frais de formation du personnel...), qui procurent aux entreprises des avantages économiques futurs, ne peuvent pas être activées à cause des conditions restrictives de capitalisation (à savoir le caractère identifiable, le contrôle...) sauf s'ils sont acquis dans le cadre d'un regroupement d'entreprise. Après la comptabilisation initiale, l'IAS 38 précise qu'une immobilisation incorporelle amortissable doit être amortie sur sa durée d'utilité selon le rythme de consommation des avantages économiques futurs. En comparaison, le référentiel comptable français comporte peu de développement sur les critères d'inscription à l'actif des éléments incorporels ainsi que sur leur traitement comptable après leur comptabilisation initiale. Il les traite généralement au cas par cas. Leur mode de comptabilisation varie entre la passation en charge, l'activation optionnelle et l'activation obligatoire. Pour les immobilisations amortissables, la durée et le mode d'amortissement ne sont pas strictement définis et laissent une marge discrétionnaire dans l'application des règles comptables.

En ce qui concerne les opérations de regroupement d'entreprise, elles font ressortir dans la majorité des cas une différence entre le coût d'acquisition et la part d'intérêt de l'acquéreur dans la juste valeur [5] La juste valeur est le montant pour lequel un actif... [5] des actifs et passifs identifiables acquis à la date de prise de contrôle (Martory et Verdier, 2000). Régie par la norme IFRS 3, la comptabilisation de cet écart d'acquisition ; appelé encore goodwill ; constitue l'un des problèmes les plus ardus en comptabilité. Les difficultés résident dans l'identification et l'évaluation des éléments du goodwill, comme la surévaluation de la firme achetée (Johnson et Kimberkey, 1998). En revanche, le goodwill généré en interne n'est pas capitalisé du fait que les dépenses qui participent à sa formation ne sont pas en pratique identifiables des dépenses courantes ou de celles nécessaires pour maintenir sa valeur. Cela crée une distorsion dans la comparabilité de firmes ayant des modes de croissance différents.

Le traitement comptable du goodwill acquis après sa comptabilisation initiale complique un peu plus la situation. Selon le PCG, il doit être amorti alors que l'IFRS 3 et l'IAS 36 recommandent d'effectuer des tests de dépréciation de valeur.

L'amortissement permet à la fois de répartir le coût d'acquisition du goodwill sur sa durée d'utilité (de consommation des avantages économiques futurs) et de faire disparaître progressivement sa valeur du bilan. On aboutit ainsi à une valeur identique à celle du goodwill généré en interne. Toutefois, l'amortissement du goodwill entraîne notamment (Jennings et alii, 1996 ; Henning et alii 2000) : 
- une dégradation systématique du goodwill ainsi qu'une durée de vie finie,

- une valeur nette du goodwill sans rapport avec la valeur économique de l'entreprise,

- des amortissements du goodwill peu représentatifs de la perte de valeur de ce dernier.

Le non-amortissement du goodwill évite ces problèmes, mais les tests de dépréciation obligent les dirigeants à faire des choix sur de nombreux paramètres qui sont autant de sources de manipulation possible.

Au final, compte tenu des difficultés à définir et évaluer les actifs incorporels, on peut s'interroger sur la capacité de ces normes IAS/IFRS à fournir à l'ensemble des parties prenantes des informations plus pertinentes sur les incorporels en général, et le goodwill en particulier. Cette étude s'intéresse donc au passage aux normes IAS/IFRS des entreprises françaises et en particulier à l'impact de l'adoption de l'IAS 38 et de l'IFRS 3 sur la qualité de l'information comptable des incorporels. Nous complétons ainsi l'étude de Bessieux-Ollier et Walliser (2007) de deux principales manières. Nous étudions tout d'abord les effets du passage aux IFRS sur le résultat net, les capitaux propres et les différentes sortes d'actifs incorporels sur un échantillon plus large de firmes, puis nous analysons l'impact de ces normes sur le cours et la rentabilité des actions des firmes concernées.

Cet article comporte trois parties. Nous commençons par une synthèse des résultats des travaux antérieurs sur le contenu informationnel des incorporels. Ensuite, nous exposons nos hypothèses de recherche dans le cadre de cette problématique de première application de ces normes. Enfin, nous testons nos modèles empiriques sur un échantillon de 120 groupes du SBF 250 et discutons les résultats avant de conclure.

\section{1 - Le contenu informationnel des incorporels}

Durant ces trois dernières décennies les chercheurs ont essayé de prouver que les investissements incorporels contribuent aux performances futures des entreprises, constituant de ce fait des actifs, et recèlent donc un certain contenu informationnel. La plupart des études s'intéressent soit aux dépenses de recherche et développement (R\&D), soit au goodwill.

\section{1 - Investissements incorporels et performance}


Les premières études sur l'impact des investissements incorporels sur la performance des firmes ont été initiées il y a plus de quarante ans aux États-Unis. La motivation de ces recherches résidait dans la démonstration de l'intérêt d'inscrire les dépenses de R\&D à l'actif du bilan (plutôt que de les passer en charge) dans la mesure où elles se traduiraient par un accroissement des performances futures. Les premières recherches de Johnson (1967) et Newman (1968) sur le contenu informationnel de la R\&D n'ont pu dévoiler aucune relation significative entre les résultats futurs et les investissements en R\&D. Le FASB a ensuite interdit, en 1974, l'activation des dépenses de R\&D (Statement $n^{\circ} 2$ ).

Cette décision et ces premiers résultats ont alors donné naissance à un courant de la littérature qui a essayé de démontrer le lien entre les investissements incorporels, limités le plus souvent aux frais de R\&D et de publicité, et la performance à venir des entreprises.

Dans cette perspective, de nombreuses études ont suivi, mettant alors en évidence la pertinence de l'inscription à l'actif de ces types de dépenses. Ainsi, Hirschey (1982) et Hirschey et Connolly (1984) montrent, qu'en moyenne, les frais de publicité et de R\&D sont liés positivement au cours boursier des entreprises américaines. Sougiannis (1994), partant de l'hypothèse que la valeur de marché est le résultat de l'actualisation des performances futures de l'entreprise, confirme les résultats précédents. Il aboutit à la conclusion que l'augmentation des dépenses de $R \& D$ entraîne des profits plus importants sur une période d'au moins sept années. Par ailleurs, il suggère que les résultats non concluants des premières études menées dans ce domaine sont dus aux techniques économétriques utilisées et à la qualité des données sur la R\&D à cette époque.

Lev et Sougiannis (1996), dans la lignée des travaux de Sougiannis (1994), établissent que les capitaux propres et les résultats sont associés significativement avec les cours et les rendements boursiers lorsque les dépenses de R\&D sont activées. De même, Aboody et Lev (1998) montrent que les coûts de développement des logiciels sont reflétés comme des actifs dans les cours boursiers des entreprises et sont significativement corrélés aux résultats futurs.

Ces résultats suggèrent donc que les dépenses de $R \& D$ sont, en moyenne, considérées par les investisseurs comme des actifs amortissables plutôt que des charges immédiates. Elles contredisent ainsi la principale raison sur laquelle se base le FASB pour interdire leur activation, à savoir l'absence de preuve de l'existence d'une relation directe entre les dépenses de $R \& D$ et les revenus futurs. 
Mais ces résultats ont été obtenus à partir d'études empiriques s'appuyant sur des données américaines. Rien ne garantit que les résultats et les relations identifiées sont transposables à d'autres environnements (Ding et alii, 2007) comme celui des pays européens et notamment la France (Casta et alii 2007) du fait des différences réglementaires, culturelles ainsi que celles relatives à la structure des marchés (Ding et alii, 2005 ; Ball et alii, 2003 ; Hope, 2003 ; Hope et alii, 2006 ; Ali et Hwang, 2000 ; Pope et Walker, 1999). La problématique relative au traitement comptable des frais de R\&D pourrait, à elle seule, illustrer cette difficulté (Casta $e t$ alii 2007) pour trois principales raisons :

- Aux États-Unis, le marché est informé du montant total des frais de R\&D (les dépenses de R\&D non capitalisées sont reportées distinctement dans le compte de résultat) alors qu'en France, généralement l'information n'existe que pour le montant capitalisé.

- À l'inverse, en France il y a une distinction entre les frais de la recherche fondamentale et les dépenses de $R \& D$ qui présentent de sérieuses chances de réussite contrairement aux États-Unis. Il en résulte que les études américaines s'intéressant à la pertinence de l'activation des dépenses de R\&D mesurent l'impact de la capitalisation de la totalité de ces dépenses.

- Les études américaines retraitent les données comptables des entreprises pour montrer leur pertinence à expliquer les données boursières en cas de capitalisation des frais de R\&D. La portée des conclusions reste assez limitée dans la mesure où s'il était réellement possible de capitaliser les dépenses de $R \& D$, il n'est pas sûr que les entreprises adoptent le même comportement en matière de gestion du résultat et de divulgation de l'information financière, et les investisseurs réagissent de la même manière aux informations publiées sur les incorporels.

En fait, les études portant sur le contexte international tendent, globalement, à confirmer les résultats américains. Ainsi, Zhao (2002), dans une étude comparative portant sur 4 pays (France, Grande-Bretagne, Allemagne et États-Unis) conclut que : (1) la divulgation de l'information relative au montant total des dépenses de R\&D augmente l'association entre la valeur de marché et les données comptables comme le résultat et les capitaux propres dans les pays où l'activation de ces dépenses est interdite (Allemagne et États-Unis), (2) l'allocation 
des dépenses de R\&D entre actif et charge augmente la pertinence de l'information comptable dans les pays qui autorisent cette pratique (Grande-Bretagne et France).

Toutefois, les études menées dans le contexte français sont en opposition avec la majorité des résultats précédents. Ding et alii (2004) ont essayé d'analyser les déterminants de l'activation des dépenses de R\&D et l'impact de ce choix sur la pertinence des données comptables des entreprises du SBF 250. Leurs résultats stipulent que les entreprises qui activent leurs dépenses de R\&D sont celles qui sont uniquement cotées sur le marché français, appartiennent au secteur de haute technologie et ont un risque Bêta plus élevé. Toutefois, leur analyse statistique ne confirme pas si cette décision améliore la pertinence des données comptables. D'un autre coté, Cazavan-Jeny (2004) montre sur la période de 1994 à 1999 que les dépenses immatérielles passées en charge ou en actif ne sont pas associées au ratio bookto-market. Ainsi, elle suggère que ces informations ne sont pas utiles pour expliquer l'écart entre la valeur comptable et de marché des firmes. De plus, l'étude de Cazavan-Jeny et Jeanjean (2005) sur 93 entreprises cotées sur la période 1998-2000 dégage une association négative entre les frais de $\mathrm{R} \& \mathrm{D}$ inscrits à l'actif et le rendement boursier. Ce résultat contredit la plupart des recherches américaines et internationales. Les auteurs expliquent ces résultats antagonistes par le fait que les dirigeants choisissent l'inscription à l'actif des dépenses de R\&D pour des raisons opportunistes ou sont incapables de discriminer les projets de $R \& D$ rentables de ceux qui ne le sont pas. Cazavan-Jeny et Jeanjean (2006) confirment ces résultats sur une période plus longue en établissent que les firmes qui capitalisent leurs dépenses de R\&D ont des rendements moins élevés et sont sous valorisées par rapport à celles qui passent ces dépenses en charge.

De manière similaire, Dufour et Zemzem (2005), essayant d'analyser les pratiques des entreprises françaises cotées sur le nouveau marché en matière de comptabilisation des dépenses de $R \& D$, stipulent que l'information comptable relative à la $R \& D$ n'est pas utilisable pour l'analyse externe. Toutefois, ils signalent que la seule variable explicative de l'activation des frais de $R \& D$ est la rentabilité. En effet, la propension à activer les frais de R\&D est plus importante chez les entreprises dont la rentabilité est faible. Cela suggère que l'activation est motivée par la volonté d'améliorer le résultat comptable, confirmant ainsi les avancées de Cazavan-Jeny et Jeanjean (2005) et (2006) au sujet de l'opportunisme des dirigeants. Ces résultats remettent en cause le bien-fondé de la capitalisation des dépenses de $\mathrm{R} \& \mathrm{D}$, comme le préconise l'IAS 38, dans un pays comme la France où le niveau de 
protection des actionnaires est relativement faible par rapport aux États-Unis (Cazavan-Jeny et Jeanjean, 2005 et 2006).

En ce qui concerne les droits de propriété intellectuelle, la littérature est plus consensuelle et indique en général un lien positif et significatif entre le nombre de brevets détenus et la capitalisation des firmes (Hirschey et alii, 2001 ; Hall et alii, 2005).

Enfin, le cas des marques a fait l'objet de peu d'études. Au Royaume-Uni, Kallupur et Kwan (2004) trouvent que les actifs incorporels identifiables, y compris les marques, sont des éléments représentant une part significative des capitaux propres des firmes (44\% en valeur médiane) et suffisamment fiables pour transmettre aux investisseurs des informations pertinentes. Mais leur fiabilité varie avec les incitations perçues par les dirigeants à reporter ce type d'information.

En France, Walliser (1999) ainsi que Stolowy et alii (2001) remarquent que les conditions d'inscription des marques à l'actif du bilan évoquées par le PCG (et précisées par le rapport sur les marques du CNC de 1992) sont très proches de celles de la norme IAS 38. On anticipe ainsi de faibles différences de comptabilisation des marques entre les normes PCG et IAS 38. En revanche, ces auteurs mettent en évidence «des différences majeures entre la France et l'Allemagne dans l'application de la norme IAS 38 pour la comptabilisation des marques ». Un autre résultat important de cette étude est que les comparaisons internationales sont à interpréter avec prudence car même lorsque des pays utilisent une norme comptable identique, les interprétations de cette norme peuvent diverger et conduire à des incidences comptables significativement distinctes.

\section{2 - Impact du changement de norme sur le goodwill}

L'adoption de l'IFRS 3 et de l'IAS 36 (pour les tests de dépréciation) a deux effets sur le goodwill: une possible réévaluation et la mise en place des tests de dépréciation qui se substituent à l'amortissement systématique.

Une réévaluation du goodwill (à la hausse ou à la baisse) devrait avoir un impact sur la rentabilité boursière en fonction de sa valeur informative. À ce sujet, deux thèses s'opposent. Albouy (2002) soutient que plus le goodwill est élevé, plus la rentabilité boursière des firmes est faible. Tandis que Jennings et alii (1996) ainsi que Vance (2006) prouvent le contraire 
dans le contexte américain. L'étude plus récente de Vance (2006) supporte l'hypothèse implicite de la norme FASB 141 (publiée en 2001) selon laquelle le goodwill est une rente générant un actif même si des disparités existent selon les secteurs d'activité. De plus, les entreprises ayant un fort goodwill dégagent une rente rapportée au total des actifs plus importante que les firmes sans goodwill dans $84 \%$ des cas.

Enfin, l'étude de Cazavan-Jeny (2004) obtient dans le contexte français des conclusions similaires. Elle met en évidence une relation positive entre le goodwill et le ratio book-tomarket. Ainsi, ce lien entre le goodwill et la valorisation boursière des firmes traduit l'intégration de cette information comptable dans les cours de bourse même si elle reflète de manière imparfaite les flux de trésorerie futurs.

En ce qui concerne la dépréciation du goodwill, les études portant sur les USA sont prépondérantes puisque les changements de mode de comptabilisation sont bien antérieurs à ceux observés en Europe.

Pour de nombreux auteurs, cette dépréciation (selon la norme SFAS 142 dont l'équivalent dans le référentiel IAS/IFRS correspond à l'IFRS 3 et l'IAS 36) devrait révéler une meilleure information que l'amortissement systématique dans la mesure où ce dernier peut sous-estimer la perte de valeur réelle du goodwill (Vance, 2008). En effet, il a observé que les entreprises ayant mis en place la SFAS 142 n'ont pas toutes déprécié leur goodwill. Pour celles ayant constaté une dépréciation de leur goodwill, elle était supérieure à l'amortissement qu'elles pratiquaient auparavant.

Les études récentes de Henning et alii (2000), Hirschey et Richardson (2002), Duangploy et alii (2005), Schultze (2005) montrent également la pertinence des tests de dépréciation de valeur du goodwill inscrit à l'actif lorsqu'il n'est pas amorti. L'utilisation de tests de dépréciation de valeur permet en particulier de passer en perte les survaleurs payées sans contrepartie. Ainsi, les capitaux propres et le résultat véhiculent respectivement une meilleure information sur la valeur de l'entreprise et sa variation de valeur.

De même, l'annonce par une firme d'une dépréciation de son goodwill se traduit par une baisse de son cours dans la mesure où les investisseurs l'interprètent comme une information négative sur les avantages économiques futurs que cet actif est censé rapporter. 
Cependant, la mise en œuvre de ces tests de dépréciation n'est pas exempte de limite. En effet, elle oblige les dirigeants des firmes à réaliser des choix discrétionnaires comme le taux d'actualisation, l'évaluation des cash flows futurs, etc. (Massoud et Raiborn, 2003, Schevin ; 2005). En particulier, il devient possible de produire des « réserves de valeur » contre la comptabilisation des pertes de valeur du goodwill acquis. Ces réserves permettent ensuite l'activation du goodwill généré en interne à concurrence du goodwill initialement comptabilisé.

À l'inverse, les tests peuvent être utilisés pour nettoyer les comptes d'entreprise à la suite d'erreur stratégique ou de changement du management (Sevin et Schroeder, 2005). Cette pratique était déjà possible avec les normes PCG via les amortissements exceptionnels. L'IFRS 3 sur cet aspect aura sûrement peu d'impact. En effet, suite à l'éclatement de la bulle spéculative sur les valeurs technologiques, Vivendi a constaté un amortissement exceptionnel de 15,7 milliards d'euros en 2001, contre 12,8 milliards d'euros pour France Télécom entre 2002 et 2004 afin de déprécier leur goodwill issu des nombreuses acquisitions opérées pendant la formation de cette bulle.

En résumé, ces études montrent que les normes IAS 38 et IFRS 3 ont globalement augmenté le contenu informationnel des incorporels avec des différences sectorielles et géographiques substantielles. Il importe donc de vérifier si leur contenu informationnel est plus pertinent dans le contexte français compte tenu des résultats contrastés observés pour ce pays.

\section{2 - Hypothèses et collecte des données}

\section{1 - Hypothèses}

Les conditions d'inscription des éléments incorporels à l'actif du bilan définies par la norme IAS 38 sont plus strictes que celles des normes françaises, notamment le règlement 99-02 [6] Règlement relatif aux règles et méthodes comptables... [6]. La norme IAS 38 impose qu'un actif incorporel soit identifiable pour le distinguer clairement du goodwill, ainsi que ses avantages économiques futurs [7] Cela exclut en autre les frais de recherche fondamentale,... [7]

Ces conditions d'activation plus restrictives définies par cette norme internationale devraient d'une part, conduire les entreprises qui les adoptent à minimiser les actifs incorporels dans les 
bilans, comme l'ont suggéré Gatet P. et Tassin H, (1998) et d'autre part, générer un phénomène de glissement des éléments incorporels non identifiables vers le goodwill, comme l'ont identifié Bessieux-Ollier C. et Walliser E. (2007) dans leur étude sur les sociétés du CAC 40. Selon ces auteurs, certains actifs incorporels tels que les parts de marché et les fonds de commerce sont reclassés en goodwill dans la mesure où ils ne répondent plus aux critères d'activation séparée exigés par le référentiel international. Dès lors, la mesure comptable du goodwill refléterait l'écart d'acquisition et un ensemble d'éléments non homogènes ne pouvant être reconnus séparément et qualifiés d'incorporels, tels que ceux identifiés par Cros I. et Sabah D. (2008) [8] Cros I. et Sabah D. (2008) ont identifié avec précision... [8], notamment les synergies, les équipes et leur savoir-faire et la capacité de la firme à poursuivre son activité à long terme.

Ces études nous conduisent à tester les hypothèses présentées ci-dessous sur un échantillon beaucoup plus large de sociétés cotées.

Hypothèse 1a: La norme IAS 38 conduit les entreprises à minimiser les actifs incorporels, autres que le goodwill, dans leur bilan.

Hypothèse $1 \mathrm{~b}$ : Le goodwill, et notamment les éléments incorporels non identifiables qu'il intègre, augmente avec le passage aux normes IFRS notamment sous l'effet conjugué de l'IFRS 3 et l'IAS 38 .

Hypothèse 1c: Le passage aux normes IFRS n'a pas fait varier de manière significative le montant global des actifs incorporels (effet de substitution entre le goodwill et les autres actifs incorporels [9] Dans la suite de l'article, précisions que les « autres... [9] ).

En outre, les données sur les actifs incorporels sont souvent utilisées par les investisseurs et les analystes financiers comme des indicateurs prédictifs de la valeur et de la performance des entreprises. Or, les états financiers en normes IAS/IFRS présentent, contrairement à ceux en normes PCG, une information détaillée sur la totalité des dépenses incorporelles capitalisées ou passées en charge dans les annexes. De plus, l'interdiction des traitements optionnels et des méthodes dérogatoires dans le référentiel IAS/IFRS ne réduirait pas seulement les risques de manipulation comptable mais, en augmentant la comparabilité des données comptables entre les firmes, rendrait de tel comportement plus facilement détectable. Ces éléments devraient réduire l'asymétrie d'information entre les dirigeants et les investisseurs, atténuer par conséquence le problème de sous-évaluation des entreprises intensives en $R \& D$ et 
augmenter ainsi l'association entre les données comptables et boursières des entreprises. Ainsi, l'hypothèse présentée ci-dessous a été testée.

Hypothèse 2a : Une information détaillée sur le contenu informationnel des actifs incorporels sous IFRS est plus pertinente qu'une information globale. En d'autres termes, dissocier le goodwill des autres éléments incorporels véhiculera une information plus pertinente sous IFRS qu'un montant global des actifs incorporels.

Toutefois, certains chercheurs signalent le fait que les normes internationales offrent un champ discrétionnaire important aux dirigeants pour apprécier les dépenses immatérielles capitalisables et déterminer la durée d'utilité des immobilisations incorporelles pour effectuer les tests de dépréciation de valeur du goodwill. Cette discrétion donnerait aux dirigeants plus de moyens pour gérer les résultats (Stolowy et Breton, 2003 et Cazavan-Jeny et Jeanjean, 2005). Il faut souligner que la latitude managériale n'est pas plus restreinte, au moins pour les incorporels, sous le référentiel PCG. D'abord, le traitement optionnel pour plusieurs dépenses immatérielles offre au dirigeant le choix d'activer ou non ces dépenses. Ensuite, en choisissant l'activation, ils peuvent manipuler le montant à capitaliser. En particulier, la propension à activer les dépenses de $R \& D$ est plus importante pour les firmes dont la rentabilité est faible (Dufour et Zemzem, 2005). Enfin, la charge d'amortissement peut être également manipulée en sous-estimant ou surestimant la durée d'utilisation des actifs car le PCG 1999 ne donne aucune règle pour la détermination de la durée d'amortissement des dépenses de R\&D capitalisées. Il la limite seulement à 5 ans.

De plus, les conditions d'activation restrictives des normes IAS/IFRS créent une certaine divergence dans le traitement de certaines dépenses (comme les marques, les parts de marché et les frais de recherches) selon qu'elles sont acquises ou produites en interne. Ainsi, les entreprises qui connaissent une croissance interne doivent passer ces dépenses en charge et devraient voir leurs données comptables perdre en pertinence par rapport aux entreprises qui croissent par des opérations de fusion acquisition. Cette situation ne joue pas en faveur de la comparabilité des données comptables.

Malgré ces différents effets contraires, on anticipe que les changements induits par les normes IAS/IFRS améliorent le contenu informationnel des actifs incorporels.

Hypothèse $2 \mathrm{~b}$ : Le goodwill et les autres éléments incorporels sous IFRS sont associés positivement à des cours de bourse. 
Hypothèse 2c: Le goodwill et les autres éléments incorporels sous IFRS sont associés positivement à des rendements plus élevés.

Compte tenu de l'échantillon choisi, les profils d'entreprise sont très disparates en taille mais également en volume d'actifs incorporels. Ces derniers représentent de $3 \%$ (Michelin) à $70 \%$ (Sanaofi-Aventis) du bilan des entreprises. Ainsi, l'impact informationnel des normes IFRS 3 et IAS 38 sur les cours de bourse et les rendements risque de dépendre de l'importance que représentent les éléments incorporels pour les firmes. Afin de vérifier cette hypothèse, nous différencions les firmes ayant une haute densité en incorporels totaux des autres. Pour être qualifiée de la sorte, elles doivent avoir une proportion en actifs incorporels, par rapport à leur bilan, supérieure à la moyenne des firmes de l'échantillon.

Hypothèse $2 \mathrm{~d}$ : Une haute densité en incorporels totaux sous IFRS a un impact positif sur les cours de bourse et les rendements.

La réglementation relative à la publication d'informations des sociétés cotées varie d'un pays à l'autre. Cuijpers R. et Buijink W. (2005) ont étudié, pour l'année 1999, les décisions volontaires des firmes, cotées et domiciliées dans l'Union européenne, d'adopter les normes américaines (US GAAP) ou les normes internationales (IAS). Les résultats de leur étude permettent de déduire que les firmes européennes ont volontairement adopté les IAS ou les US GAAP en raison de leur cotation sur un marché américain, de leurs activités internationales étendues et de leur domiciliation dans un pays où les IAS sont explicitement autorisées comme une alternative aux normes nationales. En effet, les réglementaires comptables allemands et autrichiens autorisent depuis 1998 les sociétés cotées sur les marchés anglo-saxons à appliquer uniquement les normes comptables IAS ou US GAAP [10] Ce dernier point est confirmé par l'étude de Bessieux-Ollier..._[10] (Affes et Callimaci, 2007, Bessieux-Ollier, 2006, Cabinet Mazard, 2005 et Klee, 2000). Cette autorisation est également appliquée pour les firmes suisses mais depuis 1994 (Dumontier et Maghraoui, 2006, Dumontier P. et Raffournier, 1998). En France, l'autorité de réglementation comptable incite à adopter les normes internationales avant 2005, en parallèle des normes françaises, sans pour autant autoriser une adoption anticipée. Dès lors, les sociétés françaises cotées sur les marchés anglo-saxons publient simultanément leurs états financiers consolidés en normes US GAAP ou en normes IAS/IFRS et en normes françaises. Pourtant, Ding Y. et Alii (2008) démontrent que les 199 sociétés françaises de leur échantillon (appartenant au SBF 250), cotées sur un 
marché extérieur avaient déjà tendance en 2002 à adopter une présentation alternative (ou anglo-américaine) de leur bilan consolidé en France. Dans le contexte plus précis des éléments incorporels, Obert R. (2003) souligne que les normes comptables internationales s'inspirent fortement des règles comptables britanniques et américaines, notamment pour l'activation des actifs incorporels. Dès lors, pour assurer la comparabilité et la cohérence des informations comptables, les sociétés françaises cotées sur les marchés anglo-saxons ont tendance à inscrire en charge certains actifs incorporels non identifiables tels que les frais de R\&D même pour leurs comptes préparés selon les normes françaises. Ces études nous conduisent à tester l'hypothèse suivante selon si une société est cotée ou non sur un marché anglo-saxon.

Hypothèse 3: Une cotation sur les marchés anglo-saxons réduirait de façon anticipée la volonté des sociétés de capitaliser les éléments incorporels non identifiables et aurait un impact positif sur les cours de bourse et les rendements.

\section{2 - Collecte des données et sélection de l'échantillon}

Notre échantillon est constitué de sociétés composant le SBF 250 en octobre 2005. Les données comptables en normes françaises et en normes internationales ont pu être obtenues par une consultation directe des rapports annuels publiés et une étude des états financiers consolidés publiés au BALO [11] Ceux sont généralement les sociétés qui n’ont pas communiqué... [11]. La principale difficulté a été d'identifier les firmes qui communiquent leurs états financiers en normes IAS/IFRS. Les sociétés qui clôturent leur exercice comptable à une date ultérieure à celle du 31 décembre 2004, n'étaient pas soumises à la recommandation de l'AMF de publier également leurs états financiers en normes internationales pendant la période de transition. Pour des questions de pertinence des informations, seules les sociétés qui ont publié leurs comptes consolidés en normes IAS/IFRS ont été maintenues dans notre échantillon. Les données boursières ont été extraites de la base de données Datastream.

La Constitution de l'échantillon est proposée dans le tableau 1. 


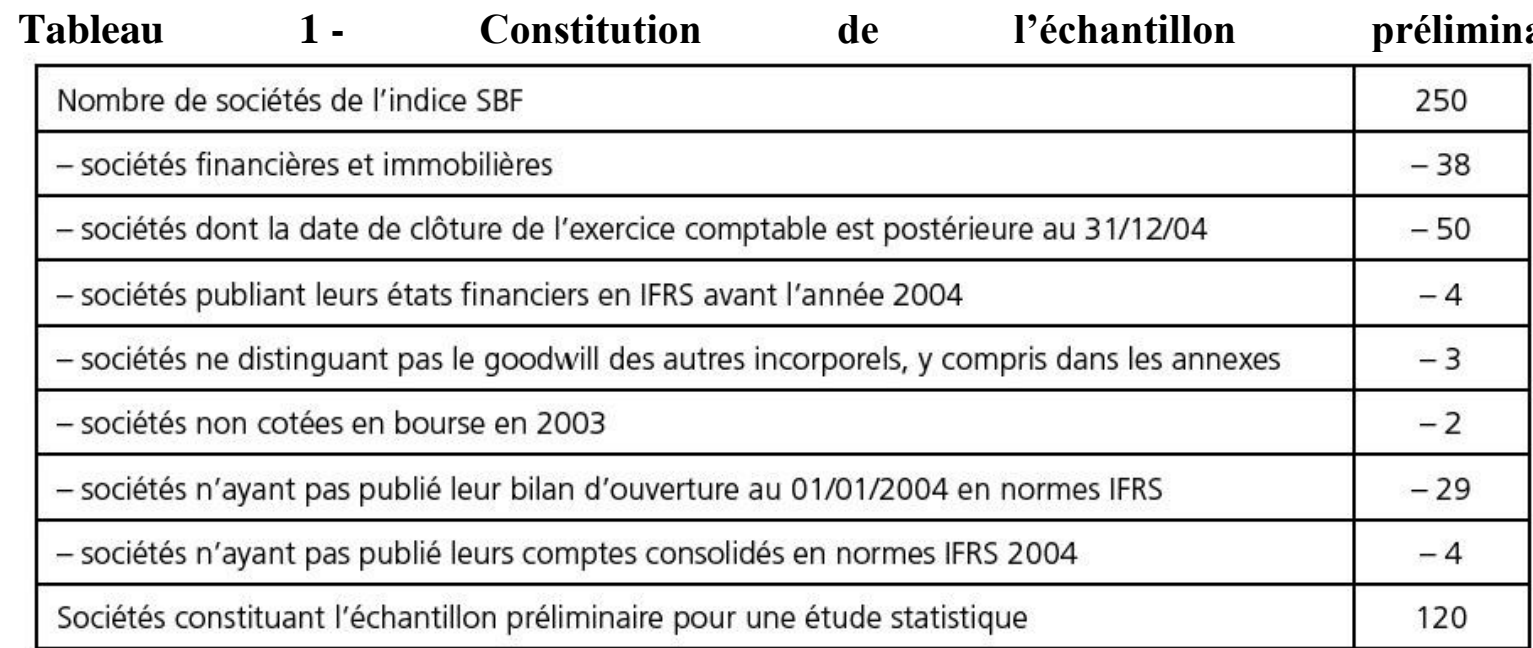

\section{3 - Méthodologie et résultats}

\section{1 - Tests univariés}

54

Avant de tester nos hypothèses de recherche 2 et 3 pour déterminer le degré de pertinence des données comptables en normes IAS/IFRS, il est essentiel d'étudier le changement éventuel de la valeur de ces données, exprimées simultanément sous deux référentiels comptables différents, à la même date, le 31 décembre 2004, sur l'ensemble de notre échantillon (hypothèses $1 \mathrm{a}, \mathrm{b}$ et $\mathrm{c}$ ). L'analyse descriptive et les résultats univariés pour l'ensemble des firmes inclues dans l'étude sont reportés dans le tableau 2.

Tableau 2 - L'impact de la transition des normes françaises vers les IFRS de $\begin{array}{lll}\text { l'échantillon } & \text { en } & 2004^{(\mathrm{a}),(\mathrm{b}),(\mathrm{c}),(\mathrm{d}),(\mathrm{e})}\end{array}$

\begin{tabular}{|l|c|l|l|l|l|l|l|l|l|l|l|l|}
\cline { 2 - 12 } \multicolumn{2}{c|}{} & \multicolumn{3}{c|}{ Normes Françaises } & \multicolumn{2}{c|}{ IFRS } & \multicolumn{2}{c|}{$\begin{array}{c}\text { Test de } \\
\text { Moyenne }\end{array}$} & \multicolumn{4}{c|}{ Test de Wilcoxon } \\
\hline$\%$ total actifs & N & Moyenne & Médiane & Moyenne & Médiane & T test & P value & Rangs & N & Z test & P value \\
\hline GW & 120 & $11,98 \%$ & $8,60 \%$ & $15,96 \%$ & $13,96 \%$ & 4,790 & 0,000 & Positifs $^{\mathrm{a}}$ & 86 & $-6,003$ & 0,000 \\
\hline INC & 120 & $9,13 \%$ & $3,52 \%$ & $5,99 \%$ & $2,31 \%$ & $-3,641$ & 0,000 & Négatifs $^{\mathrm{b}}$ & 74 & $-3,106$ & 0,002 \\
\hline INCTOT & 120 & $21,11 \%$ & $19,36 \%$ & $21,95 \%$ & $20,05 \%$ & 1,825 & 0,071 & Positifs $^{c}$ & 70 & $-2,959$ & 0,003 \\
\hline RN & 120 & $3,95 \%$ & $3,84 \%$ & $4,59 \%$ & $4,18 \%$ & 4,124 & 0,000 & Positifs $^{d}$ & 86 & $-4,675$ & 0,000 \\
\hline CP & 120 & $37,58 \%$ & $36,78 \%$ & $37,25 \%$ & $34,71 \%$ & $-0,764$ & 0,446 & Négatifs $^{\circ}$ & 69 & $-1,390$ & 0,165 \\
\hline
\end{tabular}

GW : goodwill

INC : autres actifs incoporels

INCTOT : actifs incorporels totaux

$\mathrm{RN}$ : résultat net

$\mathrm{CP}$ : capitaux propres

(a) GW normes IFRS > GW normes françaises 
(b) INC normes IFRS < INC normes françaises

(c) INCTOT normes IFRS > INCTOT normes françaises

(d) RN normes IFRS > RN normes françaises

(e) CP normes IFRS < CP normes françaises

L'adoption des normes IAS/IFRS pour l'établissement des états financiers a réellement généré des modifications dans la valeur des données comptables. Sur l'ensemble de l'échantillon, le résultat net (rn) a augmenté en moyenne de 3,95\% du total des actifs en normes françaises à $4,59 \%$ du total des actifs en normes IFRS (la médiane passant de 3,84 \% à 4,18\%). Cette hausse de $16 \%$ est significative statistiquement.

L'impact de la norme IAS 38 sur les actifs incorporels paraît plus prononcé. En effet, les immobilisations incorporelles totales [12] Précisions à nouveau, que les immobilisations incorporelles... [12] (inctot) ont augmenté, en moyenne, de près de $4 \%$ lors du passage au nouveau référentiel comptable. Plus précisément [13] L’interprétation de cette analyse est identique si... [13], le goodwill (gw) a augmenté de 11,98 \% à 15,96\% du total des actifs (la médiane croissant de $8,6 \%$ à $13,96 \%$ ), soit une différence de $33,2 \%$ statistiquement significative. À l'inverse, la moyenne des autres immobilisations incorporelles (inc) a diminué de $9,13 \%$ à 5,99\% du total des actifs (la médiane de 3,53\% à 2,31\%). Cette diminution de $34,4 \%$ est également significative statistiquement.

Selon le test de Wilcoxon [14] Lorsque la somme des rangs des différences positives... [14], plus de $71 \%$ des sociétés de notre échantillon (86 firmes sur 120) ont revu à la hausse la valeur du goodwill lors du passage aux normes IFRS et plus de $61 \%$ d'entre elles ont diminué la valeur comptable des autres immobilisations incorporelles. Le test de Wilcoxon est significatif au seuil de $1 \%$, excepté pour les capitaux propres.

Ces modifications dans la valeur des données comptables sont statistiquement significatives alors que la majorité des entreprises ont choisi de recourir de façon optionnelle à l'exemption à l'IFRS 3 et à l'IAS 38, comme l'autorise la norme IFRS 1 [15] Pour faciliter la première application des nouvelles... [15]. Demaria et Dufour (2007) confirment dans leur étude une large application de ces deux exemptions par la plupart des sociétés du SBF 120. De même, Cazavan-Jeny et Jeanjean (2007) considèrent que les firmes françaises ont majoritairement fait des choix comptables, autorisés par les exemptions de la norme IFRS 1, permettant de minimiser les effets du changement de normes. Si les sociétés de notre échantillon avaient été 
contraintes de retraiter leurs états financiers rétrospectivement, sans possibilité d'exemptions, les modifications dans la valeur des données comptables lors du passage aux normes internationales auraient été davantage significatives.

Une première interprétation de ces résultats suggère que les sociétés auraient procédé à un transfert des actifs incorporels non individualisés vers le goodwill. Soumis à des conditions d'enregistrement plus restrictives en normes IFRS, les actifs incorporels ne doivent plus intégrer des éléments non identifiables. Seuls les actifs séparables peuvent être qualifiés d'éléments incorporels. Ainsi, dans cette analyse initiale des résultats, la valeur comptable des actifs incorporels non individualisés serait intégrée avec celle du goodwill. Ce glissement des actifs incorporels non identifiables vers le goodwill tend à confirmer les hypothèses $1 \mathrm{a}$ et $1 \mathrm{~b}$.

Précisons qu'en application de l'IRFS 3 et de l'IAS 38 révisé, le goodwill et les immobilisations incorporelles à durée de vie indéterminée subissent également une revalorisation supplémentaire en raison de la suppression des amortissements obligatoires dont ils ont fait l'objet. Cette revalorisation supplémentaire du goodwill et des autres immobilisations incorporelles justifierait la hausse moyenne de seulement $4 \%$ des immobilisations incorporelles totales (inctot) de notre échantillon et confirmerait notre hypothèse $1 \mathrm{c}$.

Ces résultats confortent ceux de Bessieux-Ollier et Walliser (2007) obtenus sur les entreprises composant le CAC 40. La relative stabilité globale des actifs incorporels dans le total des actifs non courants était contrebalancée par des effets de substitution; certaines entreprises reclassant en goodwill des immobilisations incorporelles qui ne répondaient plus à la définition donnée par les normes IFRS comme les parts de marché ou encore les fonds de commerce.

Outre la constatation de ces reclassements des éléments incorporels non identifiables, inscrits à l'origine dans les autres immobilisations incorporelles, vers le goodwill, il convient de s'interroger sur la manière dont les investisseurs les perçoivent? Quel est l'impact de cette transposition sur le cours et la rentabilité des actions de la firme ? La mesure comptable du goodwill, traduisant non seulement la valeur de l'écart de première consolidation mais aussi la valeur des actifs incorporels non individualisés de façon plus conséquente sous l'influence des normes internationales, est-elle considérée comme une information plus pertinente pour les 
investisseurs ? Une analyse multivariée permettra de tester les hypothèses 2 et 3 et de proposer des éléments de réponses.

\section{2 - Tests multivariés}

\subsection{1 - Association entre les actifs incorporels et le cours boursier}

Pour déterminer la pertinence des informations comptables liées aux incorporels, en examinant leur impact sur le marché financier, nous utilisons un premier modèle, fréquemment utilisé dans la recherche empirique, qui étudie la relation entre le prix des titres (P) et la valeur comptable des capitaux propres par action ordinaire et du bénéfice par action (BPS [16] Ce modèle a été motivé par les travaux théoriques sur...[16] ). La valeur comptable des capitaux propres est décomposée en valeur comptable par action ajustée des éléments incorporels capitalisés (CPPSA) et en valeur comptable par action des actifs incorporels totaux (INCTOTPS). En outre, pour isoler la pertinence de la valeur comptable du goodwill et des autres incorporels, la valeur comptable par action des actifs incorporels totaux (INCTOTPS) est décomposée en valeur comptable par action du goodwill (GWPS) et en valeur comptable par action des autres incorporels (INCPS). Les firmes dont le total des actifs incorporels est supérieur à la moyenne [17] Les actifs incorporels totaux, en pourcentage du total... [17] de l'échantillon sont considérées comme ayant une haute densité en incorporels totaux (HDIT). De même, la cotation (C) d'une société sur les marchés anglo-saxons est intégrée dans le modèle. L'aléa $\varepsilon$ représente la partie inexpliquée du cours de l'action. Les équations (1) et (2) de ce premier modèle se présentent ainsi :

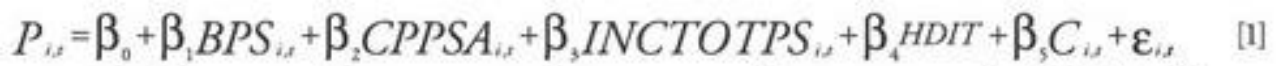

$$
\begin{aligned}
& P_{i, s}=\beta_{0}+\beta_{i} B P S_{i,}+\beta_{2} C P P S A_{i,}+\beta_{3} G W P S_{i, s}+\beta_{i} I N C P S_{i, j}+\beta_{5} H D I T+\beta_{6} C_{i,}+\varepsilon_{i, d}[2]
\end{aligned}
$$




\begin{tabular}{|c|c|}
\hline$P_{i, t}$ & $=$ prix d'une action de la firme $i 3$ mois après la date de clôture de l'exercice comptable $t$. \\
\hline$B P S_{i, t}$ & $=$ Résultat net par action de la firme $i$ de l'exercice comptable $t$. \\
\hline$C P P S A_{i, s}$ & $\begin{array}{l}=\text { Valeur comptable des capitaux propres par action de la firme } i \text { de l'exercice comptable } t \\
\text { ajustée des actifs incorporels totaux. }\end{array}$ \\
\hline$I_{N C T O T}$, & $\begin{array}{l}=\text { Valeur comptable des actifs incorporels totaux par action de la firme } i \text { de l'exercice } \\
\text { comptable } t \text {. }\end{array}$ \\
\hline$G W P S_{i, t}$ & $=$ Valeur comptable du goodwill par action de la firme $i$ de l'exercice comptable $t$. \\
\hline$I_{N C P S}$ & $\begin{array}{l}=\text { Valeur comptable des autres actifs incorporels par action de la firme } i \text { de l'exercice } \\
\text { comptable } t \text {. }\end{array}$ \\
\hline$H D I T_{i, t}$ & $\begin{array}{l}=\text { Haute densité en incorporels totaux de la firme } i \text { en fin d'année } t: \text { variable muette } \\
\text { égale à } 1 \text { si la firme } i \text { a des actifs incorporels totaux supérieurs à la moyenne de } \\
\text { l'échantillon (moyenne }=21,11 \% \text { en normes françaises et } 21,95 \% \text { en IFRS selon } \\
\text { tableau 2) et } 0 \text { dans le cas contraire. }\end{array}$ \\
\hline$C_{i, t}$ & $\begin{array}{l}\text { = Cotation sur les marchés anglo-saxons de la firme } i \text { en fin d'année } t \text { : variable muette } \\
\text { égale à } 1 \text { si l'entreprise est cotée sur le New York Stock Exchange (NYSE), le NASDAQ ou } \\
\text { le London Stock Exchange (LES) et à } 0 \text { dans le cas contraire. }\end{array}$ \\
\hline
\end{tabular}

L'information sur la valeur comptable du goodwill et des autres incorporels est disponible seulement lorsque les états financiers sont publiés, soit trois mois après la date de clôture de l'exercice comptable. De même que Aboody et Lev (1998), nous considérons que la variable dépendante sera le cours de l'action trois mois après la date de clôture de l'exercice.

Ce modèle d'évaluation présente l'avantage d'utiliser les données comptables comme une approximation des flux de trésorerie futurs actualisés espérés par les investisseurs et par là même, de la valeur de marché d'une firme.

Les équations [1] et [2] de ce premier modèle feront l'objet de deux régressions : une première avec des données comptables en normes françaises et une seconde avec des données comptables en normes IFRS.

Selon les hypothèses $2 \mathrm{~b}$ et $2 \mathrm{c}$, la valorisation du goodwill et des autres incorporels sous le nouveau référentiel comptable doit permettre d'obtenir une meilleure prévision du cours des titres. Si la qualité globale du modèle avec des données comptables en IFRS, mesurée par le $\mathrm{R}^{2}$, est meilleure que celle du même modèle avec des données comptables sous normes françaises, alors les hypothèses $2 \mathrm{~b}$ et $2 \mathrm{c}$ seront validées.

Le coefficient sur INCPS devrait être positif si le montant des éléments incorporels activés, selon les normes IFRS, a une plus forte valeur prédictive auprès des investisseurs. Le coefficient sur GWPS devrait également être positif si les investisseurs ont perçu, que sous 
IFRS, le goodwill peut intégrer des incorporels non identifiables auxquels seraient attachés des avantages économiques futurs [18] Des éléments incorporels non identifiables comme les... [18]. A contrario, il ne devrait pas être significatif s'ils perçoivent ces éléments incorporels non identifiables comme une source d'information peu pertinente.

\subsection{2 - Résultats du modèle 1}

Les résultats statistiques des régressions linéaires du modèle (1) sont présentés dans le tableau 3 .

Tableau 3 - Régression sur les cours boursiers: Normes Françaises \& Normes $\operatorname{IFRS}^{(*),(* *)}$

\begin{tabular}{|c|c|c|c|c|}
\hline & \multicolumn{4}{|c|}{ PRIX } \\
\hline & \multicolumn{2}{|c|}{ Normes françaises } & \multicolumn{2}{|c|}{ Normes IFRS } \\
\hline & [1] & [2] & [1] & [2] \\
\hline \multirow[t]{2}{*}{ BPS } & $4,647^{* *}$ & $4,921 * \star$ & $5,983 * *$ & $5,99 * *$ \\
\hline & $(4,342)$ & $(4,555)$ & $(6,843)$ & $(6,883)$ \\
\hline \multirow[t]{2}{*}{ CPPSA } & $1,236 * *$ & $1,201 * *$ & $1,025^{* *}$ & $1,03 * *$ \\
\hline & $(10,883)$ & $(10,477)$ & $(12,577)$ & $(12,552)$ \\
\hline \multirow[t]{2}{*}{ INCTOTPS } & $0,597 * \star$ & & $0,524 * *$ & \\
\hline & $(3,499)$ & & $(3,672)$ & \\
\hline \multirow[t]{2}{*}{ GWPS } & & $0,65^{* *}$ & & $0,596 * *$ \\
\hline & & $(2,591)$ & & $(3,316)$ \\
\hline \multirow[t]{2}{*}{ INCPS } & & 0,468 & & 0,314 \\
\hline & & $(1,477)$ & & $(0,901)$ \\
\hline \multirow[t]{2}{*}{ HDIT } & 15,297 & 15,54 & $15,718^{*}$ & $16,084^{*}$ \\
\hline & $(1,877)$ & $(1,823)$ & $(2,060)$ & $(2,097)$ \\
\hline \multirow[t]{2}{*}{ Cotation } & $-3,594$ & $-4,883$ & $-5,296$ & $-5,351$ \\
\hline & $(-0,408)$ & $(-0,547)$ & $(-0,652)$ & $(-0657)$ \\
\hline \multirow[t]{2}{*}{ Constante } & 10,42 & 10,517 & 6,948 & 6,816 \\
\hline & $(1,54)$ & $(1,494)$ & $(1,095)$ & $(1,071)$ \\
\hline $\mathrm{Nb}$ d'observations & 120 & 120 & 120 & 120 \\
\hline R2 & 0,879 & 0,875 & 0,899 & 0,900 \\
\hline R2 ajusté & 0,762 & 0,753 & 0,801 & 0,800 \\
\hline $\mathrm{F}$ & 77,128 & 61,448 & 96,596 & 80,172 \\
\hline Prob $>F$ & 0,000 & 0,000 & 0,000 & 0,000 \\
\hline
\end{tabular}

Valeur absolue des statistiques $t$ entre parenthèses 
(*) Statistiquement significatif à $5 \%$

(**) Statistiquement significatif à $1 \%$

La qualité de l'ajustement et la significativité globale du modèle en normes IFRS sont supérieures à celles du modèle en normes françaises. Les résultats du modèle montrent l'existence d'une relation positive et significative au seuil de $1 \%$ entre le goodwill par action et le prix de l'action. Ainsi, l'information financière véhiculée par le goodwill capitalisé paraît tout aussi fiable en normes IFRS qu'en normes françaises. En effet, le coefficient du GWPS est positif et statistiquement significatif sous les deux référentiels comptables. Même si les éléments incorporels non identifiables sont noyés dans le tout hétérogène que constitue le goodwill, la mesure comptable de ce dernier selon les normes internationales est toujours une information pertinente pour les investisseurs.

Valorisés selon les normes internationales, les autres actifs incorporels ne deviennent pas une information plus pertinente auprès des investisseurs. Le coefficient des INCPS est positif mais ne fait preuve d'aucune significativité aussi bien sous le dispositif comptable français que sous les normes IFRS $(\mathrm{p}>0,05)$. Ainsi, les actifs incorporels individualisés à l'actif du bilan des sociétés ne seraient pas une information plus pertinente pour les actionnaires, que ne le sont les actifs incorporels non individualisés transférés dans le goodwill. Les normalisateurs ne semblent pas avoir atteint leur objectif par l'application de l'IAS 38, en privilégiant la fiabilité de l'information par l'interdiction de l'inscription de plusieurs éléments incorporels non identifiables. Ces résultats réfutent partiellement l'hypothèse $2 b$.

Toutefois, les actionnaires estiment comme source d'avantages économiques futurs le fait qu'une entreprise ait une haute densité en actifs incorporels totaux, mais uniquement dans le cadre d'une valorisation en normes internationales. Le coefficient de HDIT est positif et significatif au seuil de $5 \%$, ce résultat valide ainsi l'hypothèse $2 \mathrm{~d}$.

Précisons également que les actionnaires considèrent le contenu informationnel du total des actifs incorporels (INCTOTPS), sans distinction en goodwill et autres éléments incorporels, comme pertinent. Le coefficient des INCTOTPS est positif et significatif ( $p>0,01$ ). L'hypothèse $2 \mathrm{a}$ est réfutée puisque le pouvoir explicatif du modèle est moindre avec la distinction de deux actifs incorporels plutôt qu'en considérant un niveau global des actifs incorporels. 
L'hypothèse 3 est également réfutée (coefficient non significatif). Le fait qu'une société soit cotée sur un des marchés anglo-saxons n'induit pas d'impact sur les cours de bourse.

\subsection{3 - Association entre les actifs incorporels et le rendement boursier}

Dans la continuité de ces recherches et pour confirmer la robustesse de nos résultats, comme le suggère Easton (1999), nous testons un second modèle qui relie les rendements boursiers (R) aux variations des valeurs comptables des incorporels indentifiables par action ( $\triangle \mathrm{INCPS})$ et du goodwill par action ( $\triangle$ GWPS), outre celles du bénéfice par action $(\triangle B P S)$ et des capitaux propres ajustés par action ( $\triangle$ CPPSA).

$$
\begin{aligned}
& R_{i, s}=\beta_{0}+\beta_{1} \Delta B P S_{i j}+\beta_{2} \Delta C P P S A_{i j}+\beta_{3} \Delta I N C T O T_{i, s}+\beta_{i} H D I T_{i, s}+\beta_{5} C_{i,}+\varepsilon_{i, j}[3] \\
& \left.R_{i, t}=\beta_{0}+\beta_{1} \Delta B P S_{i,}+\beta_{2} \Delta C P P S A_{i, s}+\beta_{3} \Delta G \text { WPS }_{i,}+\beta_{4} \Delta I N C P S_{i,}+\beta_{5} H D I T_{i, t}+\beta_{6} C_{i,}+\varepsilon_{i,}, 4\right]
\end{aligned}
$$

\begin{tabular}{|c|c|}
\hline$R_{i, t}$ & $\begin{array}{l}=\text { Rendement boursier de la firme } i 3 \text { mois après la date de clôture de l'exercice } \\
\text { comptable } t . R_{i, t}\left[\left(P_{i, t}+\text { Dividendes }_{i, t} / P_{i, t-1}\right]-1 \text { où } P \text { est le prix de l'action } 3 \text { mois }\right. \\
\text { après la date de clôture de l'exercice } t .\end{array}$ \\
\hline$\triangle B P S_{i, t}$ & $=$ Variation du résultat net par action de la firme $i$ de l'année $t$. \\
\hline$\triangle C P P S A_{i, z}$ & $\begin{array}{l}\text { = Variation de la valeur comptable des capitaux propres par action de la firme } i \text { en fin } \\
\text { d'année } t \text { ajustée d'actifs incorporels totaux. }\end{array}$ \\
\hline$\triangle I N C T O T_{i, t}$ & $\begin{array}{l}\text { = Variation de la valeur comptable des actifs incorporels totaux par action de la firme } i \\
\text { en fin d'année } t \text {. }\end{array}$ \\
\hline$\Delta G W P S_{i, t}$ & $=$ Variation de la valeur comptable du goodwill par action de la firme $i$ en fin d'année $t$. \\
\hline$\triangle I N C P S_{i, r}$ & $\begin{array}{l}=\text { Variation de la valeur comptable des autres actifs incorporels par action de la firme } i \\
\text { en fin d'année } t \text {. }\end{array}$ \\
\hline$H D I T_{i, t}$ & $\begin{array}{l}\text { = haute densité en incorporels totaux de la firme } i \text { en fin d'année } t \text { : variable muette } \\
\text { égale à } 1 \text { si la firme } i \text { a des actifs incorporels totaux supérieurs à la moyenne de } \\
\text { l'échantillon (moyenne }=21,11 \% \text { en normes françaises et } 21,95 \% \text { en IFRS selon } \\
\text { tableau } 2 \text { ) et } 0 \text { dans le cas contraire. }\end{array}$ \\
\hline$C_{i, s}$ & $=$ Cotation sur les marchés anglo-saxons de la firme $i$ en fin d'année $t$. \\
\hline
\end{tabular}

Avec

Afin de disposer de données conformes au modèle à estimer, une analyse préalable des résidus [19] Cette procédure de diagnostic des observations pour... [19] nous a permis de supprimer 17 observations dont les résidus dépassaient deux fois et demi l'écart-type estimé de l'aléa en valeur absolue. Pour ce second modèle, l'échantillon définitif, pour une analyse multivariée, est composé de 103 sociétés.

\subsection{4 - Résultats du modèle 2}


Les résultats du modèle (2), reportés dans le tableau 4, nous permettent de corroborer partiellement l'hypothèse 2c : l'évolution dans la valeur comptable du goodwill selon les normes internationales a une valeur informative pour expliquer les rendements boursiers. Les coefficients du $\triangle$ GWPS sont positifs et significatifs $(\mathrm{p}<0,05)$.

Cependant, cette valeur informative est inexistante pour les investisseurs aussi bien lors d'une valorisation des autres éléments incorporels en normes françaises $(p>0,418)$ que d'une valorisation en normes IFRS. Ils ne perçoivent pas les incorporels identifiables comme des sources de valeur pour la firme. La qualité globale du modèle en norme IFRS est supérieure à celle du modèle en normes françaises (le $\mathrm{R}^{2}$ ajusté augmente de $23 \%$ à 28,2 \%).

Au vu des résultats des tableaux 3 et 4 , les faibles facteurs d'inflation de la variance (VIF < 2.0) associés à de faibles écarts type des estimations des paramètres démontrent l'absence de problèmes de colinéarités [20] Les indices de conditionnement sont tous inférieurs...[20]. 
Tableau 4 - Régression sur les rendements boursiers : Normes Françaises \& Normes $\operatorname{IFRS}^{(*),(* *)}$

\begin{tabular}{|c|c|c|c|c|}
\hline & \multicolumn{4}{|c|}{ RENDEMENT } \\
\hline & \multicolumn{2}{|c|}{ Normes françaises } & \multicolumn{2}{|c|}{ Normes IFRS } \\
\hline & [3] & [4] & [3] & [4] \\
\hline \multirow[t]{2}{*}{$\triangle \mathrm{BPS}$} & $0,02766 * *$ & $0,02623 * *$ & $0,02633^{* *}$ & $0,02699 * *$ \\
\hline & $(4,197)$ & $(4,175)$ & $(4,317)$ & $(4,253)$ \\
\hline \multirow[t]{2}{*}{$\triangle$ CPPSA } & $0,006139 * *$ & $0,005104^{*}$ & 0,005244 ** & $0,005082 *$ \\
\hline & $(2,721)$ & $(2,359)$ & $(2,611)$ & $(2,415)$ \\
\hline \multirow[t]{2}{*}{$\triangle I N C T O T P S$} & $0,006002^{*}$ & & $0,006393^{* *}$ & \\
\hline & $(2,170)$ & & $(2,710)$ & \\
\hline \multirow[t]{2}{*}{$\Delta$ GWPS } & & $0,006975^{\star}$ & & $0,006908^{*}$ \\
\hline & & $(2,019)$ & & $(2,129)$ \\
\hline \multirow[t]{2}{*}{$\triangle \mathrm{INCPS}$} & & $-0,002643$ & & 0,004854 \\
\hline & & $(-0,409)$ & & $(0,868)$ \\
\hline \multirow[t]{2}{*}{ HDIT } & 0,04812 & 0,04278 & $0,07431^{*}$ & $0,09175^{\star}$ \\
\hline & $(1,273)$ & $(1,196)$ & $(2,077)$ & $(2,495)$ \\
\hline \multirow[t]{2}{*}{ Cotation } & $-0,113^{\star *}$ & $-0,103^{*}$ & $-0,07263$ & 0,0832 \\
\hline & $(-2,618)$ & $(-2,503)$ & $(-1,782)$ & $(-1,927)$ \\
\hline \multirow[t]{2}{*}{ Constante } & $0,231 * *$ & $0,244^{* *}$ & $0,214^{* *}$ & $0,237 * *$ \\
\hline & $(8,586)$ & $(9,529)$ & $(8,078)$ & $(8,694)$ \\
\hline $\mathrm{Nb}$ d'observations & 103 & 103 & 103 & 103 \\
\hline$R 2$ & 0,529 & 0,525 & 0,564 &, 569 \\
\hline R2 ajusté & 0,243 & 0,230 & 0,283 & 0,282 \\
\hline$F$ & 7,536 & 6,083 & 9,069 & 7,737 \\
\hline Prob $>F$ & 0,000 & 0,000 & 0,000 & 0,000 \\
\hline
\end{tabular}

Valeur absolue des statistiques $t$ entre parenthèses

(*) Statistiquement significatif à $5 \%$

(**) Statistiquement significatif à $1 \%$

\section{4 - Conclusion et limites}

L'objectif de cette étude est d'analyser le degré de pertinence de l'information comptable des incorporels, notamment le goodwill et les autres actifs incorporels, lors du passage aux normes internationales. L'adoption des normes IAS/IFRS a réellement généré des 
modifications dans la valeur des données comptables des entreprises du SBF 250. L'effet bien connu en termes de résultat net par les professionnels et les chercheurs est encore plus marqué pour les actifs incorporels. La relative stabilité des immobilisations incorporelles totales (4\% en moyenne) cache en fait de forts effets de substitution des autres immobilisations incorporelles (baisse de $34 \%$ ) vers le goodwill (hausse de $33 \%$ ). De nombreuses firmes ont ainsi reclassé en goodwill des immobilisations incorporelles qui ne répondaient plus à la définition donnée par les normes IFRS. Ce phénomène de glissement observé sur le marché français se situe dans la lignée des réflexions de Gatet P. et Tassin H. (1998).

Les tests empiriques démontrent l'existence d'une plus grande pertinence dans les informations véhiculées par les données comptables du goodwill et des actifs incorporels totaux valorisés selon les normes IFRS. En effet, les incorporels totaux et le goodwill sont statistiquement significatifs sous les deux référentiels comptables, au contraire des autres actifs incorporels, mais la qualité de l'ajustement et la significativité globale du modèle en normes IFRS est supérieure à celle du modèle en normes françaises.

Malgré le durcissement des critères d'inscription des actifs dans la catégorie des autres actifs incorporels (IAS 38), seul le goodwill explique les cours de bourse et les rendements lorsqu'on dissocie les types d'actifs incorporels pour le modèle en normes IFRS. Les investisseurs semblent donc continuer à ne porter d'attention qu'au goodwill qui serait toujours perçu comme un générateur de rentes. Ces résultats, qui traduisent une contribution substantielle du goodwill à la valeur actionnariale, se situent dans la même lignée que la plupart des études américaines (Jennings et alii, 1996 et Vance, 2006) et des études réalisées sur le marché français (Cazavan-Jeny, 2004). Cette première phase d'application des normes IFRS pour l'année 2004, durant laquelle les investisseurs n'auraient pas pris toute la mesure de la norme IAS 38, justifierait ce lien positif entre le goodwill et la valorisation boursière des firmes.

Ce phénomène amène à s'interroger sur d'une part la perception du goodwill par les investisseurs compte tenu des reclassements opérés lors du passage aux IAS/IFRS qui ont conduit à le faire croître de manière significative, d'autre part l'utilité pour les investisseurs de l'IAS 38 qui interdit l'inscription de plusieurs éléments incorporels non identifiables au bilan.

De plus, lorsqu'on discrimine les firmes selon leur densité en actifs incorporels, on remarque que ce critère explique leur cours et les rendements uniquement en normes internationales. 
Dans ce cadre, les actionnaires estiment ainsi une haute densité en actifs incorporels totaux comme une source d'avantages économiques futurs.

Enfin, le fait qu'une société soit cotée sur un des marchés anglo-saxons n'induit pas d'impact sur les cours de bourse, ce qui est conforme à la théorie de l'efficience des marchés, mais tend à infirmer les résultats obtenus par Cuijpers R. et Buijink W. (2005) dont l'étude a été réalisée sur une période différente (1999), reflétant un contexte boursier très différent et sur un échantillon de plus de 1700 sociétés.

Dans leur rapport annuel, la majorité des sociétés ont choisi l'option offerte par les normes IFRS 1 de ne pas retraiter les regroupements d'entreprises antérieurs à la date de transition, soit le $1^{\text {er }}$ janvier 2004. De ce fait, notre analyse comparative entre l'application des normes internationales et des normes françaises comporte une imperfection. En effet, le passage aux normes IFRS ne sera complet que pour les comptes consolidés au 31 décembre 2005. Les comptes consolidés 2004 de la plupart des sociétés de notre échantillon ont été élaborés sans application anticipée des normes IAS 32 et 39 relatives aux instruments financiers. L'évolution des cours boursiers et des rentabilités ne sont pas affectées par la juste valeur de ces instruments financiers, ce qui introduit un biais supplémentaire dans la véracité de nos résultats.

En outre, il convient de souligner que l'échantillon retenu est composé de seulement 120 sociétés du SBF 250 et repose sur des données comptables et boursières d'une seule année (2004). Réaliser cette étude sur un échantillon plus large avec une prise de recul de 3 années au moins permettrait de capturer avec plus de justesse l'impact des normes internationales, IAS 38 révisée et IFRS 3, sur les cours et les rentabilités boursières des entreprises cotées.

En définitive, on a pu constater que le goodwill selon les normes IAS/IFRS représente l'ensemble des synergies non seulement du regroupement d'entreprises mais également des éléments incorporels non identifiables dus à des reclassements induits par l'IAS 38. Ce phénomène ne risque-t-il pas de faire évoluer la perception du goodwill par les investisseurs ?

\section{Bibliographie}

EN LIGNE Aboody, D., Lev, B. (1998). The value relevance of intangibles: the case of software capitalization. Journal of Accounting Research 36 : 161-191. 
- Affes, H., Callimaci, A., (2007). Les déterminants de l'adoption anticipée des normes comptables internationales : choix financier ou opportunisme ?, Comptabilité-Contrôle-Audit 13 (2) : 149-166.

- Albouy, M. (2002), Acquisitions, «goodwill» et performance boursière. L'Expansion Management Review décembre : 72-79.

- Ali, A., Hwang L. (2000). Country-specific factors related to financial reporting and the value relevance of accounting data. Journal of Accounting Research 38 : 1-21.

- Ball, R., Robin, A., Wu, J.S. (2003). Incentives versus Standards: Properties of Accounting Income in Four East Asian Countries and Implications for Acceptance of IAS. Journal of Accounting and Economics 36 : 235-270.

- Benabdellah, S., Teller, R. (2006). Immobilisations corporelles et immeubles de placement en IFRS : le coût historique va-t-il résister à la juste valeur?, Revue Française de Comptabilité mars : 40-44.

- Besley, D.A., Kuh, E., Welsch, R.E. (1980), Regression Diagnostic, John Wiley \& Sons.

- Bessieux-Ollier, C. (2006). Les pratiques d'évaluation et de publication des entreprises françaises, allemandes et américaines : le cas des éléments incorporels. Comptabilité-Contrôle-Audit 12 (2) : 167-189.

- Bessieux-Ollier, C., Walliser, E. (2007). La transition et le bilan de la première application en France des normes IFRS: le cas des incorporels. Comptabilité-Contrôle-Audit 13 (numéro spécial) : 219246.

- Casta, J.F., Escaffre, L., Ramond, O. (2007). Investissement immatériel et utilité de l'information comptable : Étude empirique sur les marchés financiers européens. Conférence SIFF, Louvain-laNeuve, 16-17 septembre.

- Cazavan-Jeny, A. (2004). Le ratio market-to-book et la reconnaissance des immatériels - une étude du marché français. ComptabilitéContrôle-Audit 10 (2) : 99-24.

- Cazavan-Jeny, A., Jeanjean, T. (2005). Pertinence de l'inscription à l'actif des frais de R\&D : une étude empirique. Comptabilité-ContrôleAudit 11 (1) : 5-21.

- Cazavan-Jeny, A., Jeanjean, T. (2006). The Negative Impact of R\&D Capitalisation: A Value Relevance Approch. European Accounting Review 15 (1) : 37-61.

- Cazavan-Jeny, A., Jeanjean, T. (2007). Accounting choices under IFRS 1: analysis and détermiants. Third annual workshop of the European Financial Reporting Research Group, ESSEC, Paris. 
- Chan, K., Lakonishok, J., Sougiannis, T. (2001), The Stock Market Valuation of Research and Development Expenditures. Journal of finance 56 (6) : 2431-2456.

- Cros, I., Sabah, D. (2008). Comptabilisation et suivi des acquisitions : la communication financière sur l'application d'IFRS 3 et IAS 36. In Étude des états financiers 2007 des sociétés du CAC 40, PricewaterhouseCoopers.

- Cuijpers, R., Buijink, W. (2005). Voluntary Adoption of Non-local GAAP in the European Union: A study of Determinants and Consequences. European Accounting Review 14 (3) : 487-524.

- Demaria, S., Dufour, D. (2007), Les choix d'options comptables lors de la transition aux normes IAS/IFRS : quel rôle pour la prudence ?, Comptabilité Contrôle Audit 13, Numéro spécial IFRS : 195-218.

- Deng, Z., Lev, B. (2006). In-process R\&D: To capitalize or expense? Journal of engineering and technology management 23 : 18-32.

- Ding, Y., Jeanjean, T., Stolowy, H. (2005). Why do national GAAP differ from IAS? The role of culture. The International Journal of Accounting $40: 325-350$.

- Ding, Y., Jeanjean, T., Stolowy, H. (2008). The impact of firms' internationalization on financial statement presentation: Some French evidence. Advances in International Accounting 24 (1) : 145-156.

- Ding, Y., Stolowy, H., Tenenhaus, M. (2004). Les déterminants de la stratégie de capitalisation des frais de recherche et développement en France. Revue Finance Contrôle Stratégie 7 (4) : 87-106.

- Ding, Y., Stolowy, H., Tenenhaus, M. (2007). R\&D productivity: an exploratory international study. Review of Accounting and Finance 6 (1) : 86-101.

- Duangploy, O., Shelton, M., Omer, K. (2005). The value relevance of goodwill impairment loss. Bank Accounting \& Finance : 23-28.

- Dufour, D., Zemzem, A. (2005). Activation des frais de R\&D : le cas des entreprises françaises cotées sur le nouveau marché. $26^{\mathrm{e}}$ congrès annuel de l'Association Francophone de Comptabilité, Lille, 11-13 mai.

- Dumontier P., Raffournier B. (1998). Why firms Comply Voluntarily with IAS: an Empirical Analysis with Swiss Data. Journal of International Financial Management and Accouting 9 (3) : 216-245.

- Dumontier P., Maghraoui R. (2006). Adoption volontaire des IFRS, Asymétrie d'information et fourchettes de prix : l'impact du contexte informationnel. Comptabilité Contrôle Audit 12 (2) : 27-48.

- Gatet, P., Tassin, H., (1998). La norme IASC va conduire à minimiser les actifs immatériels. Analyse Financière 116 : 26-27. 
- Hall, B.H., Jaffe, A., Trajtenberg, M. (2005). Market Value and Patent Citations. The Rand Journal of Economics 36 (10) : 16-38.

- Hennings, S.L., Lewis, B.L., Shaw, W.H. (2000). Valuation of the components of purchased goodwill. Journal of Accounting Research $38: 375-386$.

- Hirschey, M. (1982). Intangible Capital Aspects of Advertising and R \& D Expenditures. The Journal of Industrial Economics 30 (4) : 375390.

- Hirschey, M., Connolly, R. (1984). R \& D, Market Structure and Profits: A Value-Based Approach. The Review of Economics and Statistics 66 (4) : 682-686.

- Hirschey, M., Richardson, V.J., Scholz, S. (2001). Value Relevance of Nonfinancial Information: The Case of Patent Data. Review of Quantitative Finance and Accounting 17 : 223-235.

- Hirschey, M., Richardson, V.J., (2002). Information content of accounting goodwill numbers. Journal of Accounting and Public Policy 21 (3) : 173-191.

- Hope, O.K. (2003). Disclosure practices, enforcement of accounting standards and analysts' forecast accuracy: An international study. Journal of Accounting Research 41 : 235-272.

- Hope, O.K., Jin, J., Kang, T. (2006). Empirical evidence on jurisdictions that adopt IFRS. Journal of International Accounting Research 5 (2) : 1-20.

- Jennings, R., Robinson J., Thompson, R.B., Duvall, L. (1996). The relation between accounting goodwill numbers and equity values. Journal of Business Finance \& Accounting 23 : 513-533.

- Johnson, J. (1967). A consequential Approach to Accounting for R\&D. Journal of Accounting Research Autumn : 164-172.

- Johnson, L.T., Kimberley, R.P. (1998). Is Goodwill an Asset? Accounting Horizons 12 : 293-303.

- Kallapur, S., Kwan, S. (2004). The Value Relevance and Reliability of Brand Assets Recognized by U.K. Firms. The Accounting Review 78 (1) : 151-172.

- Klee L., (2000). Comptabilité en Allemagne. Encyclopédie Comptabilité-Contrôle de gestion et Audit (Coord par B. Colasse), Éditions Economica, 279-290.

- Lantz, J-S., Sahut, J-M. (2005). R\&D Investment and the Financial performance of Technological Firms. International journal of Business 8 (4) : 365-384.

- Lev, B. (1999). R\&D and Capital Markets. Journal of Applied Corporate Finance 11 (4) : 21-35. 
- Lev, B., Bharat, S., Sougiannis, T (2005). R\&D Reporting Biases and Their Consequences. Contemporary Accounting Research 22 (4) : 9771026.

- Lev, B., Sougiannis, T. (1996). The capitalization, amortization, and value-relevance of R\&D. Journal of Accounting and Economics 21 (1) : 107-138.

- Lev, B., Zarowin, P. (1999). The Boundaries of Financial Reporting and How to Extend Them. Journal of Accounting Research, 37 (2) : 353-385.

- Martory, B., Verdier, F. (2000). Comment traiter le goodwill? Pratique d'une théorie, théorie d'une pratique. Comptabilité-Contrôle-Audit 6 (2) : 175-193.

- Massoud, M.F. and Raiborn, C.A. 2003. Accounting for Goodwill: Are We Better Off?, Review of Business, Spring, Vol. 24 (2), 26-32.

- Mazars (2005). Enquête européenne IFRS, dossiers Mazars, 52.

- De Montmorillon, B. (2001). L'investissement immatériel. In Images de l'investissement (Ed Charreaux G.). Paris. Vuibert, 259-282.

- Newman, M. (1968). Equating Return from R\&D Expenditures. Financial Executive, 26-33.

- Obert, R., (2003). Pratique des normes IAS/IFRS. Paris. Dunod.

- Ohlson, J.A. (2001). Earnings, Book Values, and Dividends in Equity Valuation. Contemporary Accounting Research 18 (1) : 107-120.

- Pope, P., Walker, M. (1999). International differences in the timeliness, conservatism, and classification of earnings. Journal of Accounting Research 37 : 53-87.

- Schatt, A., Gross, E. (2007). Quelle est l'incidence des normes IAS/IFRS sur les capitaux propres des entreprises françaises ? Revue Française de Comptabilité 396 : 35-39.

- Schevin, P. (2005). Les nouvelles règles en matière de dépréciation d'actifs. Revue Française de Comptabilité n ${ }^{\circ}$ 377, mai.

- Schipper, K. (2005). The Introduction of International Accounting Standards in Europe: Implications for International Convergence. European Accounting Review 14 (1) : 101-126.

- Schultze, W. (2005). The information Content of Goodwill Impairments under FAS 142: Implications for External Analysis and internal control. Schmalenbach Business Review 57 : 276-297.

- Sevin, S., Schroeder, R. (2005). Earning management: evidence from SFAS n ${ }^{\circ} 142$ reporting. Management Auditing Journal 20 (1) : 47-54.

- Sougiannis, T. (1994). The accounting based valuation of corporate R\&D. The Accounting Review 69 : 44-68. 
- Stolowy, H., Breton, G. (2003). La gestion des données comptables : une revue de la littérature. Comptabilité - Contrôle - Audit 9 (1) : 125152.

- Stolowy, H., Haller, A., Klockhaus, V., (2001). Accounting for brands in France and Germany compared with IAS 38 (intangible assets), An illustration of the difficulty of international harmonization. The International Journal of Accounting 36 (2) : 147-167.

- Vance, D.E. (2006). Is Goodwill Really an Asset? Working Paper Series WCRFS: 06-11, Rutgers Business School.

- Vance, D.E. (2008). Evidence that Companies are not Analyzing Goodwill for Impairment as Required by SFAS142, Working Paper Series WCRFS: 08-08, Rutgers Business School.

- Walliser E (1999), La mesure comptable des marques à l'heure de l'intégration européenne : une disparité préoccupante, Comptabilité Contrôle-Audit 5 (2) : 47-60.

- Zhao, R. (2002). Relative value relevance of R\&D reporting: an international comparison. Journal of International Financial Management and Accounting 13 : 153-174.

\section{Notes}

$[1]$

Preliminary Views on an improved Conceptual Framework for Financial Reporting: The Objective of Financial Reporting and Qualitative Characteristics of Decision-useful Financial Reporting Information, July 2006.

http://www.iasb.org/NR/rdonlyres/4651ADFC-AB83-4619-A75A4F279C175006/0/DP_ConceptualFramework.pdf

\section{[2]}

«Sa nouvelle structure organisationnelle devrait être opérationnelle dès l'année prochaine, et est animée de la ferme volonté de faire des normes IAS un ensemble exhaustif de normes comptables de la plus haute qualité, destinées à être utilisées sur les marchés de capitaux du monde entier. » Source : Communication de la Commission au Conseil et au Parlement européen, Stratégie de l'UE en matière d'information financière: la marche à suivre, document final du 13.6.2000, COM (2000) 359.

[3]

Cette norme a été approuvée en juillet 1998 et révisée en mars 2004. Elle prescrit le traitement comptable des immobilisations incorporelles qui ne sont pas spécifiquement traitées par d'autres normes et s'applique aux dépenses de $R \& D$, de publicité, de formation et d'établissement. 
Actif qui peut être vendu sans céder d'autres éléments du patrimoine de l'entreprise.

$[5]$

La juste valeur est le montant pour lequel un actif pourrait être échangé, ou un passif éteint, entre parties bien informées et consentantes et agissant dans des conditions de concurrence normales (NC 38, § 8).

$[6]$

Règlement relatif aux règles et méthodes comptables applicables aux comptes consolidés $[7]$

Cela exclut en autre les frais de recherche fondamentale, de formation et de publicité ainsi que les marques.

$[8]$

Cros I. et Sabah D. (2008) ont identifié avec précision ces éléments non homogènes dans leur étude des états financiers 2007 des sociétés du CAC 40. Ces auteurs font partie du département Évaluation \& Stratégie de PricewaterhouseCoopers.

[9]

Dans la suite de l'article, précisions que les « autres actifs incorporels » seront définis comme le total des immobilisations incorporelles moins le goodwill.

[10]

Ce dernier point est confirmé par l'étude de Bessieux-Ollier (2006) mais également par l'enquête européenne sur les IFRS du cabinet Mazars en avril 2005. En outre, selon cette enquête, les entreprises allemandes appartenant au Prime Standard, soit moins de la moitié des sociétés cotées, ont été légalement obligées de publier leurs comptes en IFRS ou en US GAAP dès 2003.

\section{[11]}

Ceux sont généralement les sociétés qui n’ont pas communiqué leurs états financiers en normes internationales dans leur rapport annuel, qui ont nécessité une recherche au BALO des données comptables en normes IFRS.

\section{$[12]$}

Précisions à nouveau, que les immobilisations incorporelles totales sont constituées du goodwill et des autres incorporelles.

[13]

L'interprétation de cette analyse est identique si les données comptables sont exprimées par action et non plus en pourcentage du total des actifs.

[14]

Lorsque la somme des rangs des différences positives est supérieure à la somme des rangs des différences négatives, les valeurs des données comptables telles que le résultat net, les immobilisations incorporelles totales et le goodwill, exprimés en pourcentage du total des 
actifs et valorisées selon les normes internationales, sont supérieures à celles évaluées en normes françaises.

\section{$[15]$}

Pour faciliter la première application des nouvelles normes internationales, l'IASB a publié la norme IFRS 1 Première adoption des normes IFRS qui permet de simplifier certains retraitements comptables rétrospectifs. L'IFRS 1 permet de recourir de façon optionnelle à certaines exemptions à d'autres normes du référentiel. Concernant l'exemption à l'IFRS 3, « un premier adoptant peut décider de ne pas appliquer rétrospectivement l'IFRS 3 à des regroupements d'entreprises passés » (IFRS $1, \S 15$ et B1). Pour ce qui est de l'exemption à l'IAS 38, «une entité peut décider d'évaluer une immobilisation incorporelle à la date de transition aux IFRS à sa juste valeur et utiliser cette juste valeur comme coût présumé » (IFRS $1, \S 16$ à 19) ; Compte tenu de la complexité de l'évaluation des actifs incorporels, l'IASB n'a pas encouragé leur réévaluation selon l'IAS 38.

\section{[16]}

Ce modèle a été motivé par les travaux théoriques sur les modèles d'évaluation (Ohlson, 2001).

\section{[17]}

Les actifs incorporels totaux, en pourcentage du total de l'actif, sont en moyenne de $21,11 \%$ en normes françaises et de $21,95 \%$ en normes IFRS. Les entreprises dont les actifs incorporels totaux sont supérieurs à la moyenne seront considérées comme faisant preuve d'une haute densité en actifs incorporels et inversement.

\section{[18]}

Des éléments incorporels non identifiables comme les marques, les parts de marché, etc. [19]

Cette procédure de diagnostic des observations pour identifier les points atypiques a été complétée par l'étude des diagrammes des résidus standardisés.

\section{[20]}

Les indices de conditionnement sont tous inférieurs à 5, soit largement en dessous de la limite critique fixée à 30 (Besley, Kuh et Welsch, 1980). 